\title{
Multiband Microwave Imaging Analysis of Ionosphere and Troposphere Refraction for Spaceborne SAR
}

\author{
Fan Zhang, Guojun Li, Wei Li, and Wei Hu \\ College of Information Science \& Technology, Beijing University of Chemical Technology, Beijing 100029, China \\ Correspondence should be addressed to Fan Zhang; zhangf@mail.buct.edu.cn
}

Received 28 February 2014; Revised 14 April 2014; Accepted 22 April 2014; Published 20 May 2014

Academic Editor: Wen-Qin Wang

Copyright (C) 2014 Fan Zhang et al. This is an open access article distributed under the Creative Commons Attribution License, which permits unrestricted use, distribution, and reproduction in any medium, provided the original work is properly cited.

\begin{abstract}
Ionosphere has different stratification at the different height. Troposphere has different refractivity at the different height. When microwave signals transmit through the ionosphere and the troposphere, the real propagation path is not an ideal straight line, but a slightly curved straight line. For the synthetic aperture radar (SAR) system, the actual distance errors will result in phase errors, which impact range section and azimuth section of SAR raw data. Consequently, the imaging precision has been decreased by imprecise slant range history. In this paper, we simulate the propagation path between satellite and the target according to Snell's law and analyze how the ionospheric and tropospheric refraction impact the spaceborne SAR imaging performance at L-band and $\mathrm{X}$-band. The simulation results show that the two refraction effects should be compensated in low frequency band for better image focusing performance.
\end{abstract}

\section{Introduction}

As the microwave signals transmit between SAR and targets, the imaging results are inevitably affected by the ionosphere and troposphere. The ionizing is at the height of $70 \mathrm{~km}$ to $500 \mathrm{~km}$ from the ground. Low frequency signal often suffers ionosphere influences severely, such as dispersion, Faraday rotation, refraction, rotation of polarization caused by irregularities, and so on [1-4]. For example, the ionized ionosphere induces Faraday rotation (FR) that affects radar polarization and causes signal path delays, and all of these can be ignored at high frequencies $(10 \mathrm{GHz})$ [5]. These effects all have strong relationship with the TEC (total electron content). Observations show that the electron density of the ionosphere presents a structure of stratification. Generally, we call the electron concentration distribution with height as the electron density profile. In recent years, there has been an increasing interest in the analysis of the impact of propagation link on low frequency and wide-bandwidth spaceborne SAR imaging [5]. Chen et al. put forward an operation mode for topside ionospheric sounding based on spaceborne high frequency SAR (HF-SAR) [6]. Theoretical analysis indicates that the azimuth resolution can be improved to tens of meters. Liu et al. develop a numerical model to investigate the SAR image degradation caused by an inhomogeneous ionosphere [7]. Both horizontal and vertical structures of the ionosphere are considered in this model. Although they do not study a method to compensate for the ionospheric effects on SAR, the numerical model they have developed is useful for testing different mitigation techniques. And they develop two numerical models about the ionosphere acting on the propagation characteristics of the spaceborne SAR [8]. One of the models simulates the ray-bending effects on the SAR system. Their results show substantial image shift due to this effect at $P$-band or lower frequency band. Wang et al. prensent the analytical study and numerical simulation to investigate the ionospheric effects on SAR imaging with Gaussian white noise at $P$-band [9]. They use the IRI 2001 model to generate the electron density profile and use the two-parameter spectrum to generate the horizontal electron density profile, which are also used in this paper. 
Troposphere is under the altitude of about $12 \mathrm{~km}$. It is the closest layer to the ground and a mixture of kinds of gases (nitrogen, oxygen, carbon dioxide, and so on) and water vapor. The dielectric properties of the troposphere vary with time and space. So the microwave signals transmit in the troposphere differently from in the vacuum [10]. The effect of troposphere on SAR imaging is usually ignored for that the early moderate resolution imaging would not be affected by the relative minor tropospheric path error. Consequently, there are few thorough analysis and validation related to the troposphere in SAR field. Currently, the spaceborne SAR imaging resolution has reached half a meter level. So the troposphere environmental factor has become one of the factors that cannot be ignored on spaceborne SAR imaging with high resolution. Sun et al. introduce the impact of atmospheric refraction on the high resolution airborne SAR which mainly reflects on azimuth resolution [10]. The changes of atmosphere refractive index in troposphere with the Hopfield refractive model are analyzed, and the calculation of the apparent range between radar and ground targets is given. The algorithm mentioned in paper has certain reference value to the performance and imaging analysis of the high resolution airborne SAR system which is level of centimeter. The refraction effects are addressed with assuming a horizontally nonhomogeneous troposphere [11]. The impact of the range refractivity on a terrestrial radio link is investigated in terms of radio horizon range. By acquiring simultaneous weather radar data over the test site, it is possible to flag affected SAR images and exclude them from the procedure.

Through the above introduction, the index of refraction will vary with the height both in ionosphere and troposphere [12]. According to Snell's law, the propagation of radio waves in the multilayer medium will be bent and not straight as we expected. The path error will cause many problems in SAR imaging. Therefore, we will simulate and analyze the impact of the multiband microwave propagation errors caused by the ionosphere and troposphere on SAR imaging in the following contents.

In this paper, we focus on the propagation path's bending caused by ionosphere and troposphere refraction, which are seldom synthetically considered before. First, we discuss the effects of ionospheric and tropospheric refraction on SAR imaging. In Section 2, we introduce the background of the ionospheric and tropospheric refractive model. The structure of ionosphere can be analyzed from two aspects: vertical profile and horizontal profile. In Section 3, we use the path tracing method to calculate the actual distance according to Snell's law. In Section 4, we perform SAR imaging simulation with the path errors and analyze the imaging performance. In Section 5, we draw a conclusion and put forward some plans for future research.

\section{Atmosphere Structure}

As Figure 1 shows, troposphere is at the height of 0 to $12 \mathrm{~km}$. The layer between $60 \mathrm{~km}$ and $90 \mathrm{~km}$ is the D region and

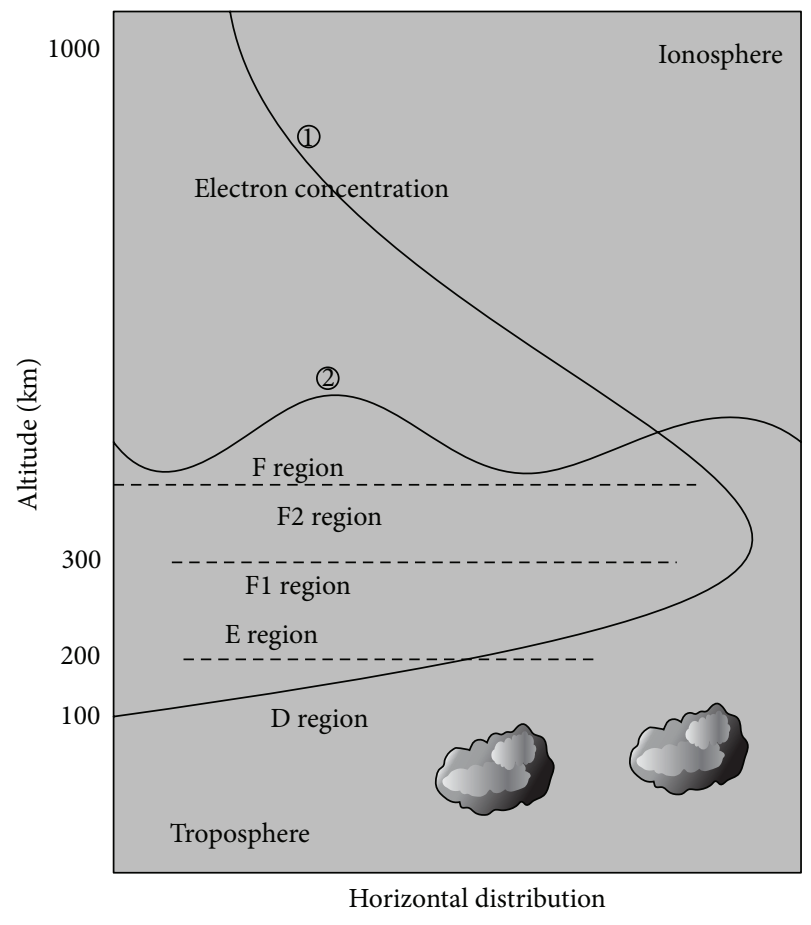

FIGURE 1: Sketch map of ionosphere and troposphere.

from $90 \mathrm{~km}$ to $140 \mathrm{~km}$ is the $\mathrm{E}$ region. The $\mathrm{F}$ region is at the height above $140 \mathrm{~km}$. In Figure 1, curve (1) represents the profile of vertical electron density; curve (2) represents the profile of horizontal electron density. Because the sketch map is just used to indicate the atmosphere structure, the vertical and horizontal electron density profile are rough. We can see that the electron concentration varies with different heights and different horizontal distribution, which will lead to the change of the ionosphere refraction. Moreover, there are kinds of gases in the troposphere, and the tropospheric index of refraction will vary with different heights as well. So there must be path errors in the propagation of microwaves.

2.1. Basic Structure of Ionosphere. The basic structure of ionosphere can be discussed from two aspects: vertical TEC profile and horizontal TEC profile.

2.1.1. Vertical TEC Profile. According to Appleton-Hatreek, we can get the simplified formula about the refractive index of ionosphere $[13,14]$ :

$$
n=\sqrt{1-\frac{80.6 N_{e}}{f^{2}}}
$$

where $N_{e}$ is the electron concentration and $f$ is the wave frequency in $\mathrm{Hz}$. 
In this paper, we use the engineering ionospheric electron density profile model IRI 2001 to calculate $N_{e}[15,16]$. The model is expressed as below [17]:

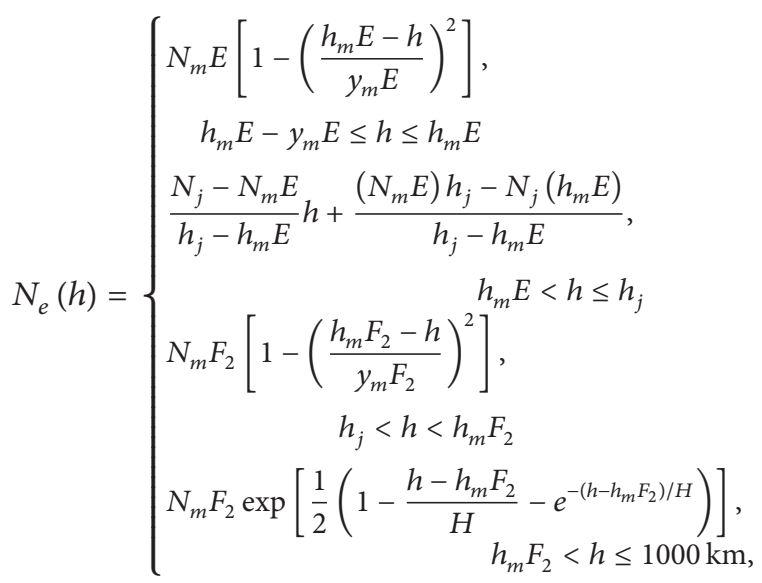

where $N_{m}$ is the largest electronic concentration of each layer, $N_{j}$ is for $F_{1}$ layer, $h$ is the height corresponding to the largest electronic concentration of each layer, and $h_{j}$ is for $F_{1}$ layer. $y_{m}$ is the half-thickness for each layer, $f_{0}$ is the critical frequency for each layer, and $f_{j}$ is for $F_{1}$ layer. $h_{m} E$ is $115 \mathrm{~km}$; $y_{m} E$ is $20 \mathrm{~km}[18]$.

The vertical electron concentration is shown in Figure 2.

2.1.2. Horizontal TEC Profile. As shown in Figure 3, the horizontal TEC varies with the different latitudes and longitudes. Since the spaceborne SAR aperture size is at least tens of kilometers, the horizontal TEC gradient will impact the SAR imaging performance.

In this paper, we use the two-parameter spectrum to generate the horizontal profile of electron concentration $[19$, 20]. $V(k)$ can be expressed as [7]

$$
V(k)= \begin{cases}\frac{\pi A}{v_{1}-1} \frac{1}{\left(k^{2}+k_{0}^{2}\right)^{v_{1}-1}} & \\ -\frac{\pi A}{\left(k_{b}^{2}+k_{0}^{2}\right)^{v_{1}-1}}\left(\frac{1}{v_{1}-1}-\frac{1}{v_{2}-1}\right), & k \leq k_{b}, \\ \frac{\pi A\left(k_{b}^{2}+k_{0}^{2}\right)^{v_{2}-v_{1}}}{v_{2}-1} \frac{1}{\left(k^{2}+k_{0}^{2}\right)^{v_{2}-1}}, & k>k_{b},\end{cases}
$$

where $k_{b}=2 \pi / L_{b}, k_{0}=2 \pi / L_{0}, k_{b}$ is the interrupt wave number, $L_{0}=10 \mathrm{~km}, L_{b}=500 \mathrm{~m}$, and $2 v_{1}=3.5,2 v_{2}=5.5$.

To generate the horizontal profile of electron concentration, a sequence of normally distributed random numbers is used in the phase of the profile spectrum. Using the discrete Fourier transform, the relationship between the electron density profile and the profile spectrum is described in the following equations:

$$
f(x)=\frac{1}{L} \sum_{n=N / 2}^{N / 2-1} F\left(k_{n}\right) \exp \left(i k_{n} x\right),
$$

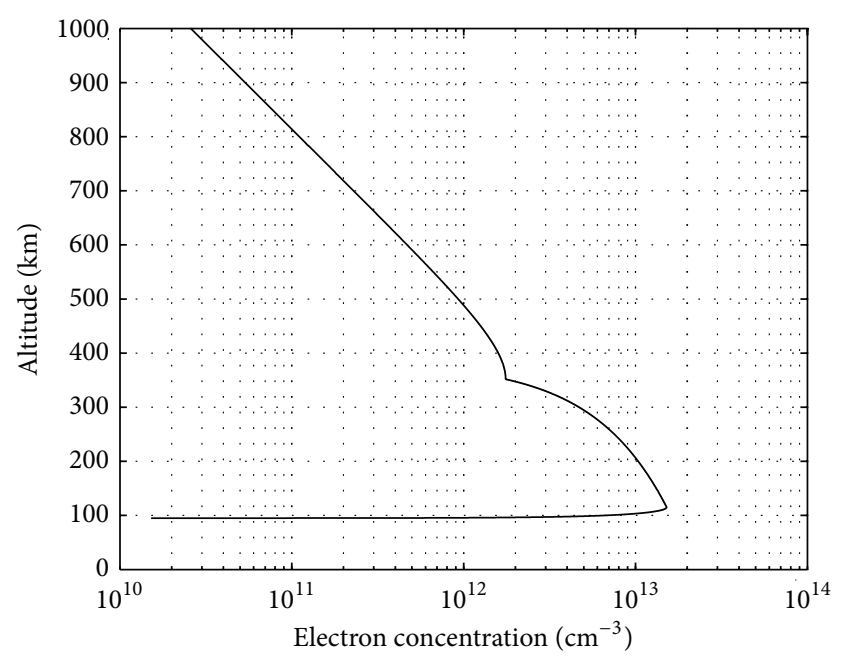

FIGURE 2: Vertical electron density profile model.

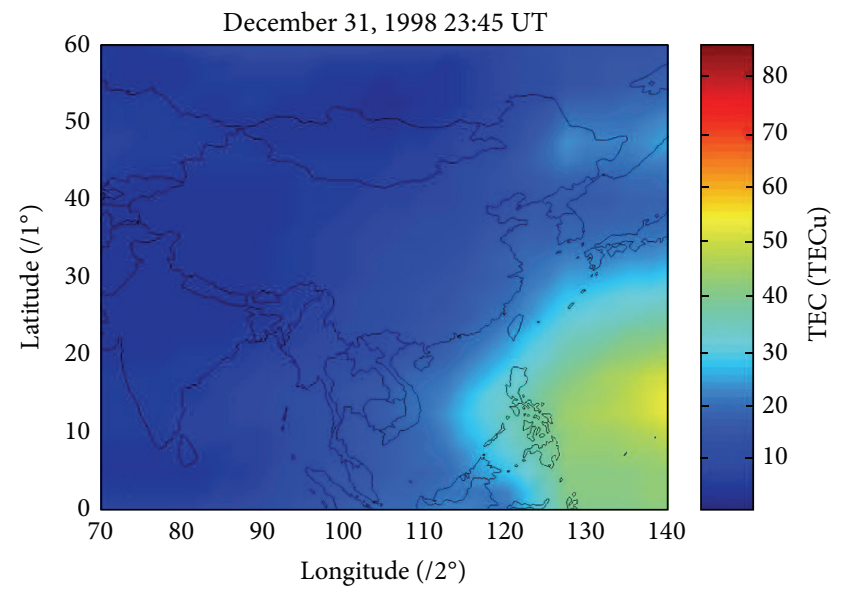

FIGURE 3: Horizontal electron density profile model.

where

$$
\begin{aligned}
& F\left(k_{n}\right) \\
& \quad=\sqrt{2 \pi L V\left(k_{n}\right)} \begin{cases}\frac{1}{\sqrt{2}}[N(0,1)+i N(0,1)], & n \neq 0, \frac{N}{2} \\
N(0,1), & n=0, \frac{N}{2}\end{cases}
\end{aligned}
$$

and $K_{n}=2 \pi n / L, L$ is a total length of a profile. $N(0,1)$ denotes a sequence of normally distributed numbers in $[0,1]$ with zero mean and unity standard deviation. The horizontal electron concentration is shown in Figure 4.

2.2. Model for Tropospheric Refractivity. As for troposphere, we often use the statistical model of the refractive index. In this paper, we use the Hopfield model [12], which includes mainly two parts. The first one is the so-called wet part, which is related to the concentration of water vapor. The other part is the so-called dry part, which is related to the gaseous nature 


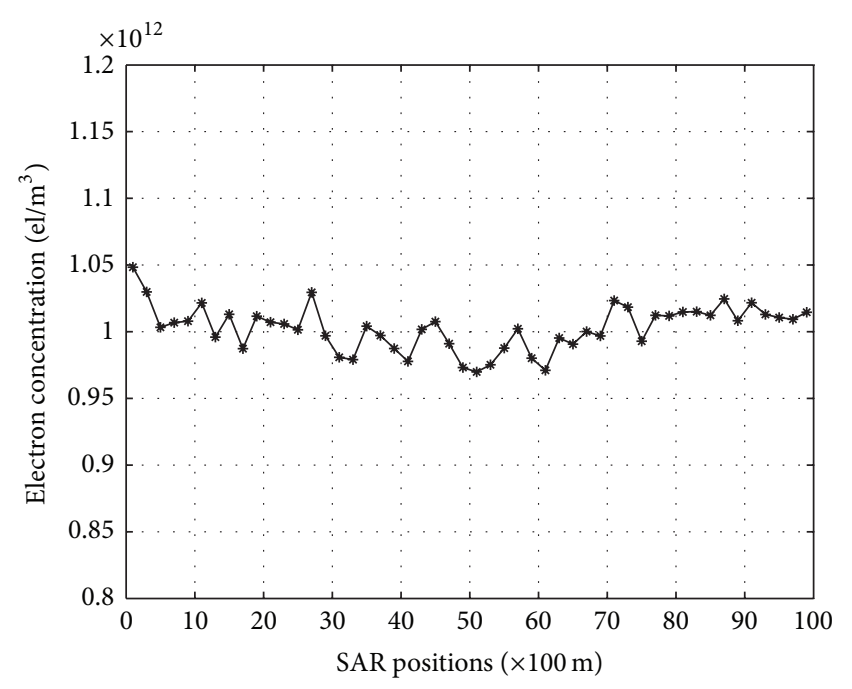

FIGURE 4: The propagation path of microwave signals.

of the lower part of the atmosphere. The dry parts and the wet parts are in form of function of fourth power [21].

$$
\begin{gathered}
N(h)=N_{d}+N_{w} \\
N_{d}= \begin{cases}N_{0 d}\left(\frac{H_{d}-h}{H_{d}-h_{0}}\right)^{4}, & h<H_{d} \\
0, & h \geq H_{d}\end{cases} \\
N_{w}= \begin{cases}N_{0 w}\left(\frac{H_{w}-h}{H_{w}-h_{0}}\right)^{4}, & h<H_{w} \\
0, & h \geq H_{w}\end{cases}
\end{gathered}
$$

where the subscripts $d$ and $w$ represent dry and wet parts; $N_{0 d}$ and $N_{0 w}$ are the ground atmospheric refractivity; $H_{d}$ and $H_{w}$ are the height where the dry and wet section of atmospheric refractivity decay to $0 ; h_{0}$ is the height of ground.

Then, we can calculate the ground atmospheric refractivity as below:

$$
\begin{gathered}
N_{0 d}=77.6 \frac{P_{0}}{\left(t_{s}+273.15\right)} \\
N_{0 w}=3.37 \times 10^{3} \frac{R H \times a}{\left(t_{s}+237.15\right)^{2}} \exp \left(\frac{b t_{s}}{c+t_{s}}\right) .
\end{gathered}
$$
by (6).

Then, we can calculate the refractive index of troposphere

\section{Calculation of the Path Error}

When the microwave signals transmit through the ionosphere and troposphere, the path will become bent because of refraction as Figure 5 shows. We can calculate the actual distance according to Snell's law. Assuming that the radar situated at a height of $h$, radar's depression angle is $\theta_{0}$,

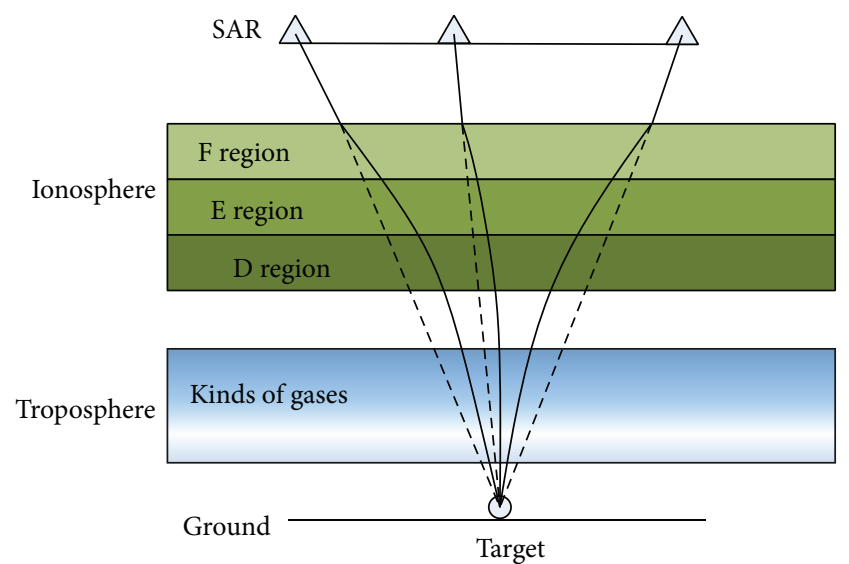

FIGURE 5: Path tracing course.

the refraction index at the height of $h$ is $n_{0}$. Then, the actual distance of the radar and the target is

$$
R=\int_{0}^{h} \frac{n^{2}(z)}{\sqrt{n^{2}(z)-n_{0}^{2} \cos ^{2} \theta_{0}}} d z .
$$

In (10), $n(z)$ is the refractive index of space, including ionosphere and troposphere. Due to the fact that different models are used, we independently calculate the actual distance in ionosphere and troposphere refraction. Furthermore, the ionospheric refraction includes two parts: vertical TEC section and horizontal TEC section.

Accordingly, the horizontal distance between radar and the target is $y_{0}$ :

$$
y_{0}=\int_{0}^{h} \frac{n_{0} \cos \theta_{0}}{\sqrt{n^{2}(z)-n_{0}^{2} \cos ^{2} \theta_{0}}} d z .
$$

According the equations above, we use the method of path tracing to calculate the actual distance of radar and the target. First, we substitute $y_{0}$ into (11) as a known quantity; thus we can get radar's depression angle $\theta_{0}$. Then, we substitute the $\theta_{0}$ into (10), and get the actual distance of radar and the target. The flowchart of the course of path tracing is expressed in Figure 6.

SAR imaging geometry is shown as Figure 7. Spaceborne SAR is at the height of $h$ and moves at a speed of $v_{a}$ along the $x$-axis direction; the instant time is $t_{m}$. When it comes to the synthetic aperture center, $t_{m}=0$. The shadow is the footprint, the horizontal width of the beam is $\beta$, and the vertical width is $\varphi$. At the $t_{m}$ moment, the actual distance between SAR and the target is $R\left(t_{m}\right)$.

If the ionospheric and tropospheric environment can be ignored, the actual distance is equal to the theoretical distance between SAR and the targets, which can be calculated as [22]

$$
R\left(t_{m}\right)=\sqrt{h^{2}+r_{0}^{2}+\left(v_{a} t_{m}\right)^{2}}
$$

So the path error is

$$
\Delta R\left(t_{m}\right)=R\left(t_{m}, n(z)\right)-R\left(t_{m}\right) .
$$




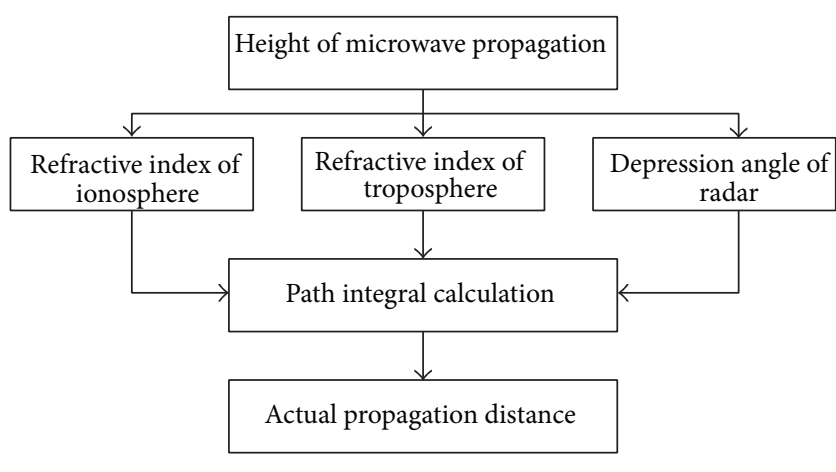

FIGURE 6: SAR imaging geometry.

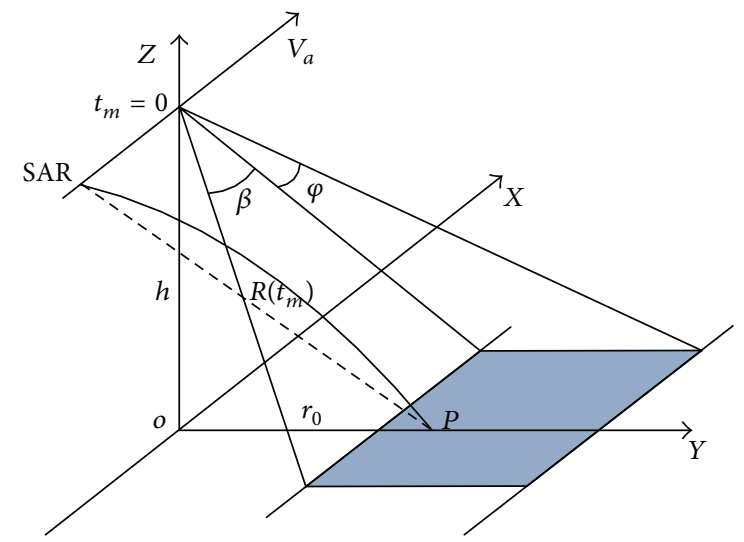

Figure 7: An example of horizontal TEC map over China and adjacent regions.

The phase error caused by path error is

$$
\Delta \varphi\left(t_{m}\right)=\frac{-4 \pi \Delta R\left(t_{m}\right)}{\lambda} .
$$

Assuming that the total synthetic aperture time is $T_{a}$, the time of range section is $\tau$ and the radar signal is chirp signal. We can get the echo that the radar receives from the target as below:

$$
\begin{aligned}
s\left(t_{m}, \tau\right) & =\sigma w_{a}^{2}(\theta) \operatorname{rect}\left(\frac{\tau-2 R\left(t_{m}\right) / c}{T_{a}}\right) \\
& \cdot \exp \left(-j \frac{4 \pi}{\lambda} R\left(t_{m}\right)\right) \cdot \exp \left(j \pi K\left(\tau-\frac{2 R\left(t_{m}\right)}{c}\right)^{2}\right),
\end{aligned}
$$

where $t$ is the azimuth slow time, $\tau$ is the range fast time, $K$ is chirp rate, $w_{a}(\theta)$ is the azimuth antenna illumination envelop, $\sigma$ is the backscatter coefficient, $c$ is the speed of light, and $R$ is the distance from antenna phase center to the target.

The echo signals that the radar receives from the target are related to the actual distance between the radar and the target. So the path error will lead to the delay and the offset error in range section.
On the other hand, we can do quadratic Taylor expansion on the actual distance $R\left(t_{m}, n(z)\right)$ around $t_{c}=r_{0} / v_{a}$ :

$$
\begin{aligned}
R\left(t_{m}, n(z)\right) \approx & R\left(\left(t_{m}-t_{c}\right), n(z)\right)+\Delta t * R^{\prime}\left(\left(t_{m}-t_{c}\right), n(z)\right) \\
& +0.5 \Delta t^{2} * R_{c}^{\prime \prime}\left(\left(t_{m}-t_{c}\right), n(z)\right)^{2} .
\end{aligned}
$$

In (16), there is a quadratic term. There must be quadratic phase error in azimuth section when the distance errors are considered. Obviously, the quadratic phase error will lead to the elevated sidelobe energy, thus affecting the SAR image quality. In summary, the path error will lead to two problems in SAR imaging: the offset error in range section and quadratic phase error in azimuth section, which can be illustrated as Figure 8.

\section{Multiband Spaceborne SAR Imaging Simulation with Refraction Path Error}

To analyze the refraction effect of atmosphere on spaceborne SAR imaging, the propagation path errors should be introduced into echo generation process in every transmitted pulse. On the other hand, the multiband responses with atmospheric refraction effects are also the key topic, which can not only guide the course of spaceborne SAR system design, but also assist the in-orbit microwave imaging and analysis. Therefore, the multiband spaceborne SAR imaging simulation is carried out for atmospheric refraction error analysis and modeling.

According to classical SAR simulation process, the imaging simulation with path error involves four steps, including refractive index calculation, propagation path error calculation, raw data simulation, and imaging analysis, as shown in Figure 9. The simulation method is as follows.

(i) According to the description of Section 2, we can calculate the different height's refractive index in ionosphere and troposphere by the general engineering model. Then we change the microwave frequency to get the different refractive index distribution along the vertical height, which can be used in actual path calculation.

(ii) Based on the path tracing method, the microwave propagation path under Snell's law is calculated with considering the ionosphere and troposphere refraction. The specific algorithm is introduced in Section 3. On this basis, we simulate the satellite orbit and calculate the path error at each pulse transmitting time and then get the error curve along the azimuth direction. To compare the multiband characteristic, it is assumed that the multiband simulation should be executed with same orbit and radar parameters. After multifrequency replacement, the multiband path error can be simulated.

(iii) The spaceborne SAR raw data simulation includes four steps: target deployment, orbit calculation, path error injection, and raw data calculation. In order to 


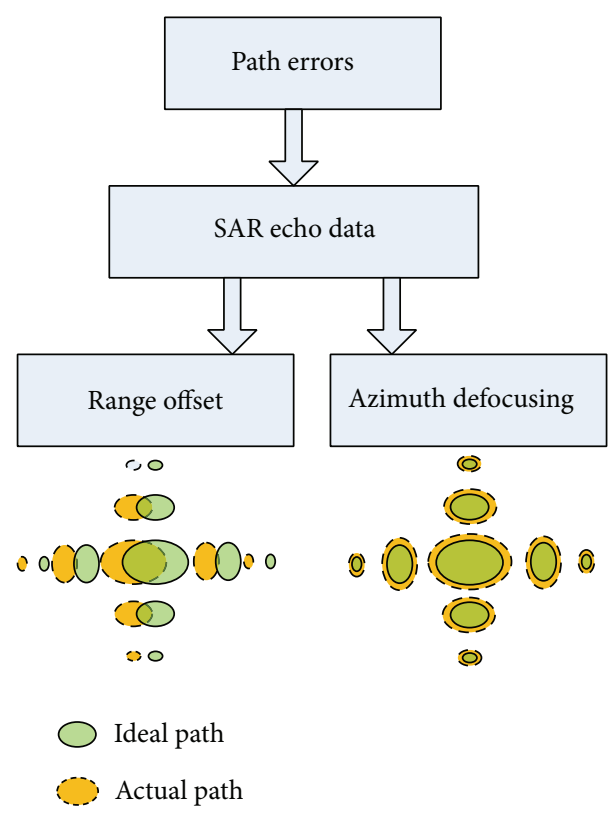

FIGURE 8: Sketch map of the offset error imaging in range section and quadratic phase error in azimuth section.

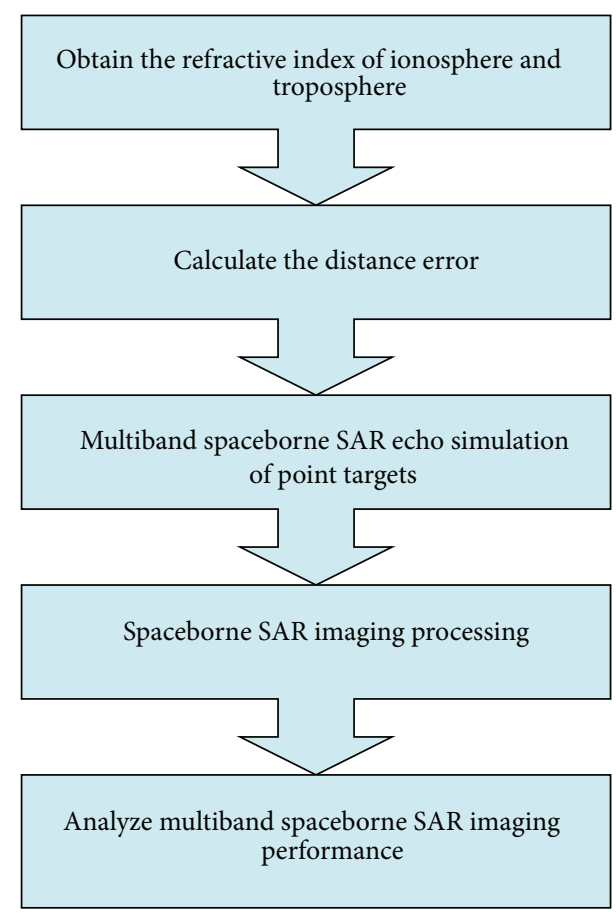

Figure 9: Process of the simulation.

measure the image quality, point targets are located in the simulated scene. Moreover, the time-domain algorithm that can easily introduce system errors is selected for spaceborne SAR raw data calculation. Through the simulation processing, the multiband SAR raw data can be generated for imaging analysis. (iv) The imaging analysis includes image processing and image quality assessment. First, the raw data should be processed by classical Chirp-Scaling imaging algorithm. Second, the point target data should be cut from SAR image for extracting the profile of range direction and azimuth direction. Finally, the SAR imaging index, such as resolution, expansion ratio, peak sidelobe ratio, and integral sidelobe ratio, can be calculated to analyze the multiband atmosphere refraction effects.

\section{Simulation Result and Discussion}

Simulations are carried out for $L$-band and $X$-band, which are applied for different remote sensing purpose and less discussed. The simulation parameters are shown in Table 1 and experimental conditions are described below:

(1) point target array is placed in the middle of simulated image;

(2) in the course of refractive index calculation, the ionosphere height is $100 \sim 1000 \mathrm{~km}$, the troposphere height is $0 \sim 12 \mathrm{~km}$, and the vertical divided layer scale of ionosphere and troposphere is, respectively, $0.1 \mathrm{~km}$ and $0.001 \mathrm{~km}$;

(3) in horizontal electron density profile model, the average peak value is set to $10^{12} \mathrm{el} / \mathrm{m}^{3}$ and the perturbation is set to $10 \%$. The simulated horizontal distance of electron density profile is $10 \mathrm{~km}$ with sampling interval of $100 \mathrm{~m}$.

Figure 10 shows the microwave propagation path errors of $L$-band and $X$-band in a synthetic aperture time. With the change of slant range, the path error also varies in quadratic curve. If we do not take the horizontal TEC into consideration, the curve will be smooth. However, the curve shows a zigzag line by considering the refraction. From the results, the path error of $L$-band is greater than $X$-band, but the two bands have the similar fluctuation. Because ionosphere is higher than troposphere, the ionosphere contributes the majority of path errors for longer propagation path. It is seen that the higher frequency band radar works with the greater the refraction effects and worse image quality. Figure 11 depicts, respectively, the point target response profile of range direction and azimuth direction in $L$-band and $X$-band. The azimuth profile in $L$-band has a serious quadratic phase error, which leads to the sidelobe uplift. The azimuth profile in $X$ band is close to the ideal curve. Otherwise, both the two range profiles are very standard. We can see that the path errors will lead to the decline in image quality indicators of azimuth section. Tables 2 and 3 provide a quantitative proof of the conclusion.

As shown in Table 2, compared with theoretical indicators, the resolution errors at $L$-band are $0.0246 \mathrm{~m}$ bigger than $X$-band in range direction and $1.9193 \mathrm{~m}$ more in azimuth section. Otherwise, the expansion ratio, the peak sidelobe ratio, and the integral sidelobe ratio are the same situations that the effects in $L$-band are worse than $X$-band, and the indicators in azimuth direction are worse than range 


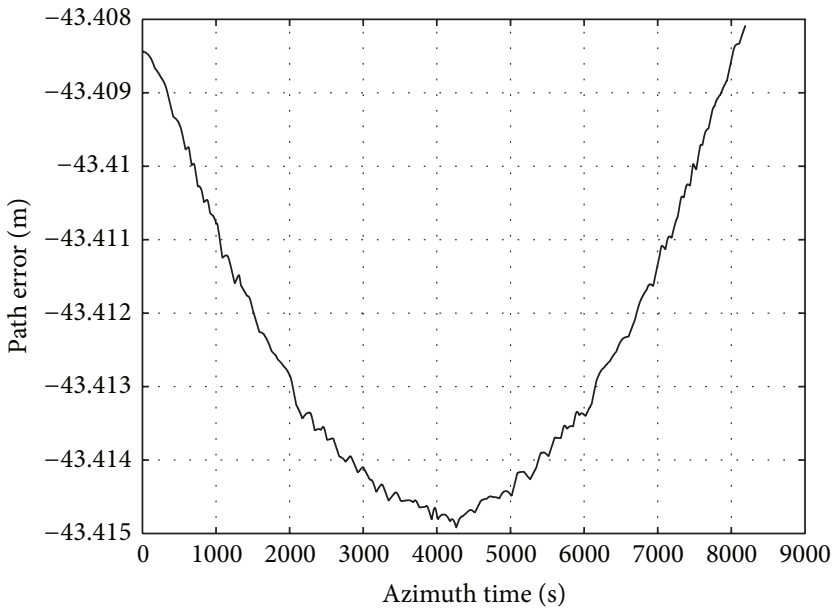

(a) $L$-band, $f=1.5 \mathrm{GHz}$

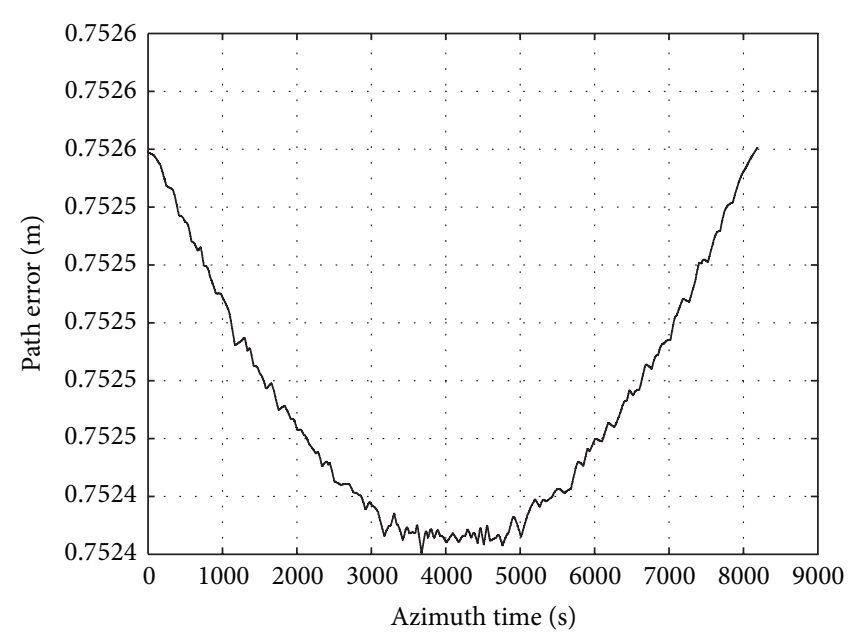

(b) $X$-band, $f=9.6 \mathrm{GHz}$

Figure 10: The path error of $L$-band and $X$-band.

TABLE 1: Simulation parameters.

\begin{tabular}{lc}
\hline Parameters & Value \\
\hline Semimajor axis & $7000 \mathrm{~km}$ \\
Eccentricity & 0.0 \\
Orbital inclination & 95 degrees \\
RAAN & 150 degrees \\
Argument of perigee & 210 degrees \\
PRF & $2000 \mathrm{~Hz}$ \\
Band width & $60 \mathrm{MHz}$ \\
Pulse width & $30 \mathrm{us}$ \\
Sampling rate & $66.6 \mathrm{MHz}$ \\
\hline
\end{tabular}

TABLE 2: Imaging indicators errors of $L$-band and $X$-band compared to the theoretical indicators in range section.

\begin{tabular}{lcc}
\hline Indicators & $L$ & $X$ \\
\hline Resolution $(\mathrm{m})$ & 0.0327 & 0.0081 \\
Expansion ratio $(\mathrm{dB})$ & 0.071 & 0.0017 \\
Peak sidelobe ratio $(\mathrm{dB})$ & 0.1072 & -0.0625 \\
Integral sidelobe ratio $(\mathrm{dB})$ & 0.2509 & -0.1326 \\
\hline
\end{tabular}

TABLE 3: Imaging indicators errors of $L$-band to $X$-band compared to the theoretical indicators in azimuth section.

\begin{tabular}{lcc}
\hline Indicators & $L$ & $X$ \\
\hline Resolution $(\mathrm{m})$ & 1.9672 & 0.0479 \\
Expansion ratio $(\mathrm{dB})$ & 0.4837 & 0.0118 \\
Peak sidelobe ratio $(\mathrm{dB})$ & -1.6258 & -0.8447 \\
Integral sidelobe ratio $(\mathrm{dB})$ & 4.6385 & -0.5886 \\
\hline
\end{tabular}

direction. As shown in Table 4, the pixel positions of the point target at $L$-band and $X$-band are different from the theoretical position. It can be seen that the lower frequency imaging is seriously affected by the propagation.
TABLE 4: Position of the point target in range section.

\begin{tabular}{lcc}
\hline Theoretical position & $L$-band & $X$-band \\
\hline$(2048.00,512.00)$ & $(2031.00,512.00)$ & $(2047.00,512.00)$ \\
\hline
\end{tabular}

According to the analysis of Section 3, the path errors also affect the position of point target. Therefore, we measure the position of two band SAR images in pixel. As can be seen from Table 4 , the range direction offset is 17 pixels and 1 pixel in $L$ band and $X$-band compared with theoretical position in the image. The image offset is proportional to the path error.

The simulation results show that path error caused by ionospheric and tropospheric refraction affects both range section and azimuth section imaging indicators especially on azimuth section, such as resolution, expansion ratio, peak sidelobe ratio, and integral sidelobe ratio. And the lower the frequency is, the more serious the effects are. Table 4 shows that there is offset error imaging in range section. With the distance between the satellite and target increasing, the imaging results will get worse. The accurate estimation of ionosphere and troposphere model will assist the autofocus compensation to improve spaceborne SAR image quality and reduce the dependence on external calibration measurement.

\section{Conclusions}

In this paper, we use the engineering practical ionospheric electron density profile model to get the electron concentration of different heights. After that, we can get the ionospheric refractive index of different heights. As for troposphere, we use Hopfield model to get the tropospheric refractive index. The actual distance is calculated by path tracing method. Compared to the propagation path in the vacuum, the real path must be bent. The path error can lead to phase error which is the key to the spaceborne SAR imaging. After researching related literatures, we find that people care a lot about the effect of troposphere and ionosphere on SAR 

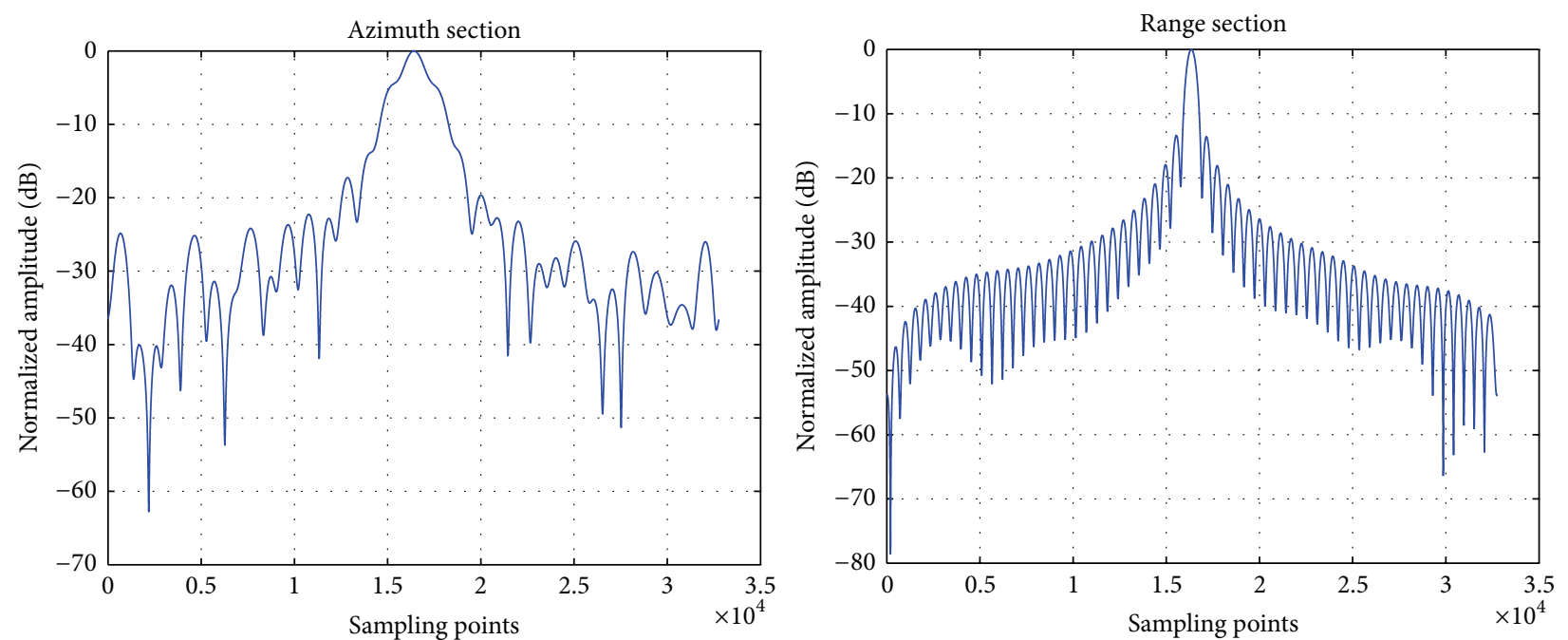

(a) $L$-band, $f=1.5 \mathrm{GHz}$
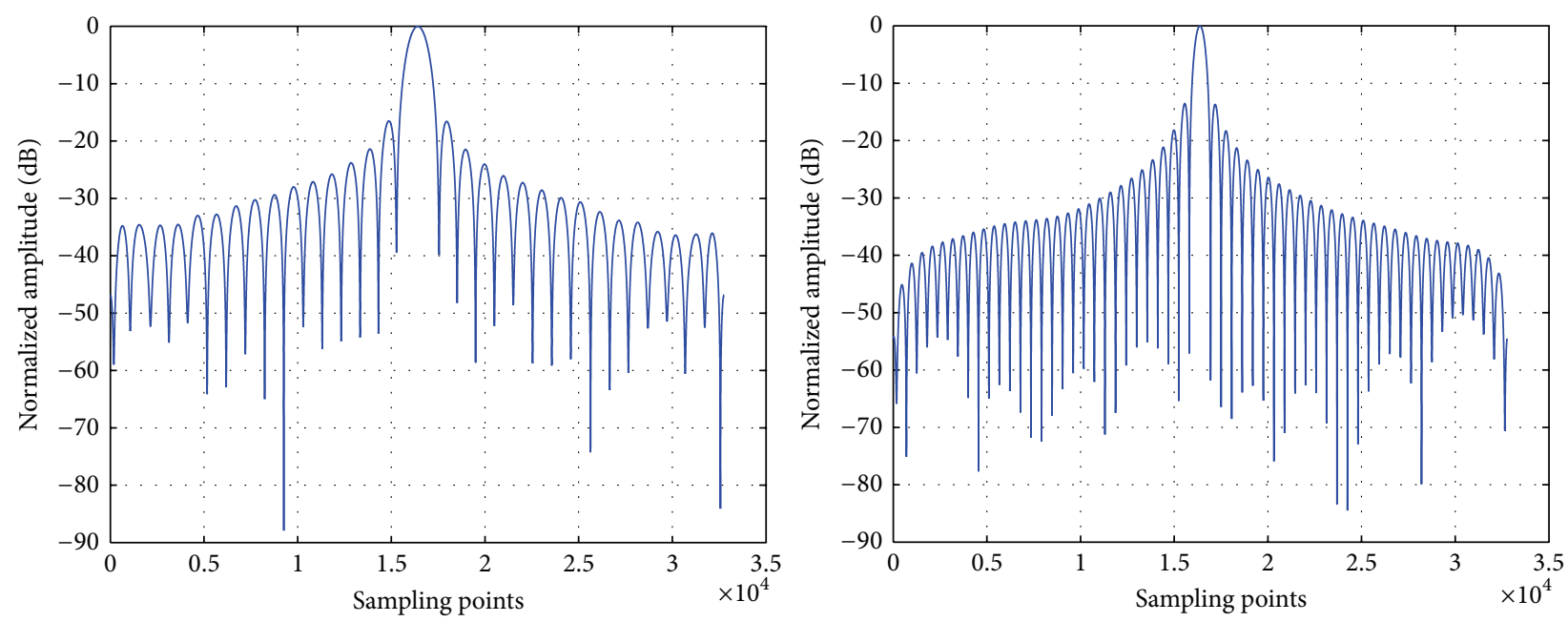

(b) $X$-band, $f=9.6 \mathrm{GHz}$
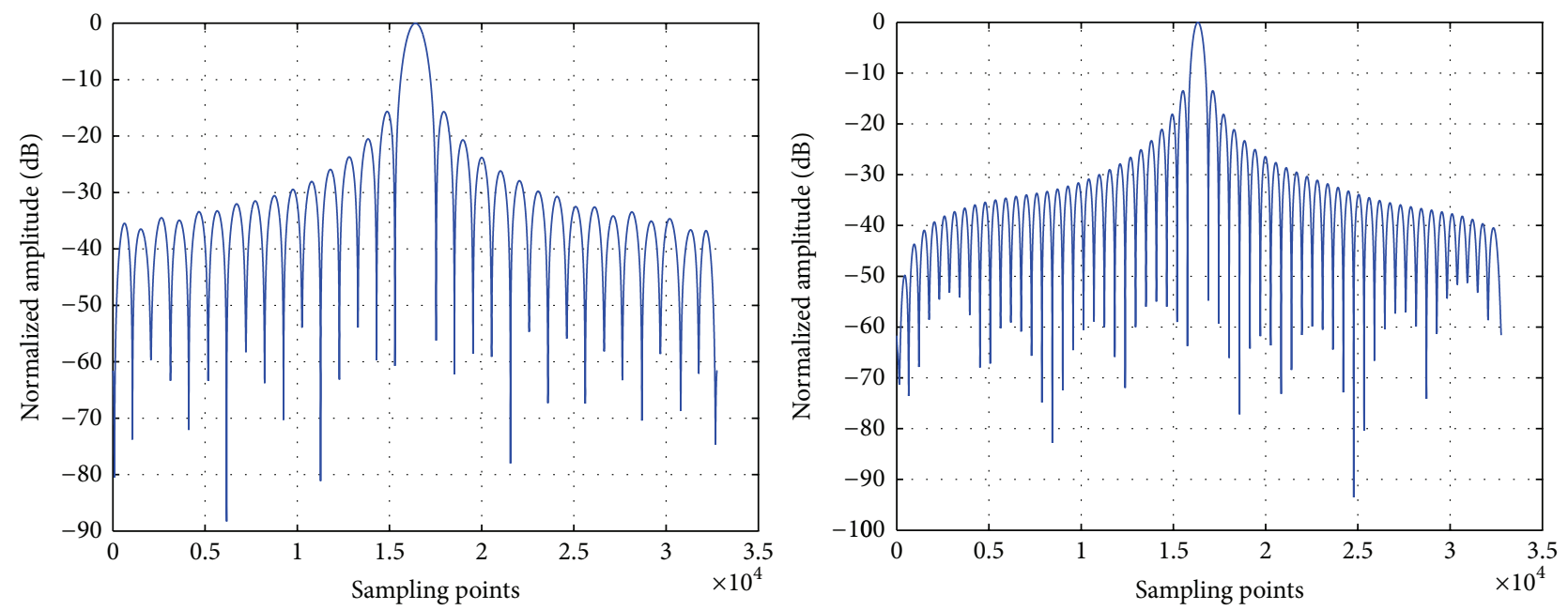

(c) Theoretical image

Figure 11: Point target assessment curve at $L$-band and $X$-band. 
imaging independently, but few materials considering both of them. So the study of the effects of two kinds of refractions on the spaceborne SAR imaging performance is essential. Other imaging indicators like image quality analyzing still needs to be further studied. In the future, we will analyze the correction of refractive effects on spaceborne SAR imaging.

\section{Conflict of Interests}

The authors declare that there is no conflict of interests regarding the publication of this paper.

\section{Acknowledgments}

This work was supported in part by the National Natural Science Foundation of China under Grant 61003132, 61302164, by the Beijing Higher Education Young Elite Teacher Project under Grant YETP0500, and by the Interdisciplinary Research Project in Beijing University of Chemical Technology. The authors sincerely thank the geological and geophysical data sharing platform of the Institute of Geology and Geophysics, Chinese Academy of Sciences for providing ionospheric TEC data contour maps of Chinese region.

\section{References}

[1] D. P. Belcher and N. C. Rogers, "Theory and simulation of ionospheric effects on synthetic aperture radar," IET Radar, Sonar \& Navigation, vol. 3, no. 5, pp. 541-551, 2009.

[2] W. S. Zhai and Y. H. Zhang, "Simulation and correction of ionospheric effects on P-band spaceborne SAR imaging," Journal of Test and Measurement Technology, no. 5, pp. 459-464, 2008 (Chinese).

[3] O. V. Goriachkin and D. D. Klovsky, "Some problems of realization spaceborne SAR in P, UHF, VHF bands," in Proceedings of the IEEE International Geoscience and Remote Sensing Symposium (IGARSS '99), vol. 2, pp. 1271-1273, July 1999.

[4] A. Danklmayer, B. J. Doring, M. Schwerdt, and M. Chandra, "Assessment of atmospheric propagation effects in SAR images," IEEE Transactions on Geoscience and Remote Sensing, vol. 47, no. 10, pp. 3507-3518, 2009.

[5] M. Jehle, M. Ruegg, L. Zuberbuhler, D. Small, and E. Meier, "Measurement of ionospheric faraday rotation in simulated and real spaceborne SAR Data," IEEE Transactions on Geoscience and Remote Sensing, vol. 47, no. 5, pp. 1512-1523, 2009.

[6] J. Chen, W. Liu, Y. Zhou, and C. Li, "Operation mode for topside ionospheric sounding based on space-borne high frequency synthetic aperture radar," in Proceedings of the 1st Asian and Pacific Conference on Synthetic Aperture Radar (APSAR '07), pp. 1-5, November 2007.

[7] J. Liu, Y. Kuga, A. Ishimaru, X. Pi, and A. Freeman, "Ionospheric effects on SAR imaging: a numerical study," IEEE Transactions on Geoscience and Remote Sensing, vol. 41, no. 5, pp. 939-947, 2003.

[8] A. Ishimaru, Y. Kuga, J. Liu, and T. Freeman, "Ionospheric effects on synthetic aperture radar at $100 \mathrm{MHz}$ to $2 \mathrm{GHz}$," Radio Science, vol. 34, no. 1, pp. 257-268, 1999.

[9] C. Wang, M. Zhang, Z. W. Xu, and C. Chen, "Simulation of ionospheric effects on SAR imaging with noise at P-band," in
Proceedings of the 10th International Symposium on Antennas, Propagation \& EM Theory, pp. 419-422, October 2012.

[10] J. P. Sun, Y. P. Wang, W. Hong, and S. Y. Mao, "Effects of atmospheric refractivity on high resolution airborne SAR performance," Journal of Systems Engineering and Electronics, vol. 34, no. 4, pp. 681-685, 2012 (Chinese).

[11] P. Valtr and P. Pechac, "The influence of horizontally variable refractive index height profile on radio horizon range," IEEE Antennas and Wireless Propagation Letters, vol. 4, no. 1, pp. 489491, 2005.

[12] P. N. Jiao and Z. Z. Zhang, Radar Environment and Transmitting Characteristic of Electric Wave, Publishing House of Electronics Industry, Beijing, China, 2004, (Chinese).

[13] M. Jehle, O. Frey, D. Small, and E. Meier, "Measurement of ionospheric tec in spaceborne sar data," IEEE Transactions on Geoscience and Remote Sensing, vol. 48, no. 6, pp. 2460-2468, 2010.

[14] J. Liu, Y. Kuga, and A. Ishimaru, "Simulations of ionospheric effects on SAR at P-band," in Proceedings of the IEEE International Geoscience and Remote Sensing Symposium (IGARSS '99), vol. 3, pp. 1842-1844, July 1999.

[15] Z. Meng and G. Y. LIU, "Ionosphere effects on the range performance of P-band spaceborne SAR signal," Modern Radar, vol. 34, no. 2, pp. 8-11, 2012 (Chinese).

[16] Z.-W. Xu, J. Wu, and Z.-S. Wu, "Potential effects of the ionosphere on space-based SAR imaging," IEEE Transactions on Antennas and Propagation, vol. 56, no. 7, pp. 1968-1975, 2008.

[17] J. M. Goodman and J. Aarons, "Ionospheric effects on modern electronic systems," Proceedings of the IEEE, vol. 78, no. 3, pp. 512-528, 1990.

[18] GJB/Z87-97, Handbook of Refraction and Attenuation of Radiowave Propagation for Radar, People's Republic of China National Military Standard, 1997, (Chinese).

[19] D. Xia, Y. Li, W. Hong, and Y. Wu, "An approach for ionospheric effects reducing on SAR imaging," in Proceedings of the 2nd Asia-Pacific Conference on Synthetic Aperture Radar (APSAR '09), pp. 1072-1075, October 2009.

[20] D.-K. Xia, Y. Li, Y.-L. Qi, W. Hong, and Y.-R. Wu, "Investigation on ionospheric decorrelation of space-borne POL-in-SAR systems," Acta Electronica Sinica, vol. 20, no. 6, pp. 1309-1314, 2011 (Chinese).

[21] J. L. Porcello, "Turbulence-induced phase errors in syntheticaperture radars," IEEE Transactions on Aerospace and Electronic Systems, vol. 6, no. 5, pp. 636-644, 1970.

[22] J. Chen, Z. Li, W. Liu, C. Li, and Y. Zhou, "Image formation algorithm for topside ionosphere sounding with spaceborne HF-SAR system," in Proceedings of the IEEE International Geoscience and Remote Sensing Symposium (IGARSS '08), vol. 2, pp. II549-II552, July 2008. 

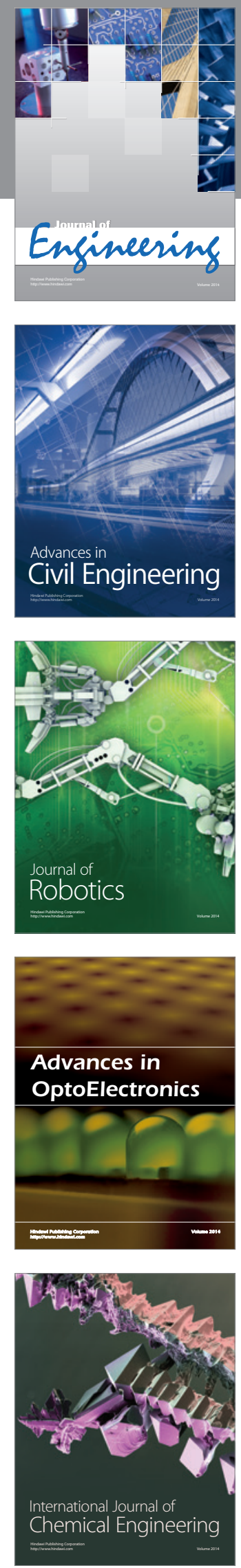

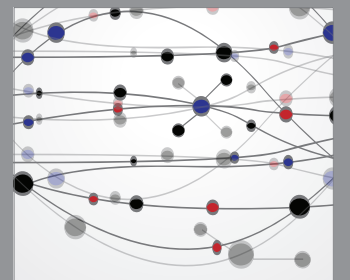

The Scientific World Journal
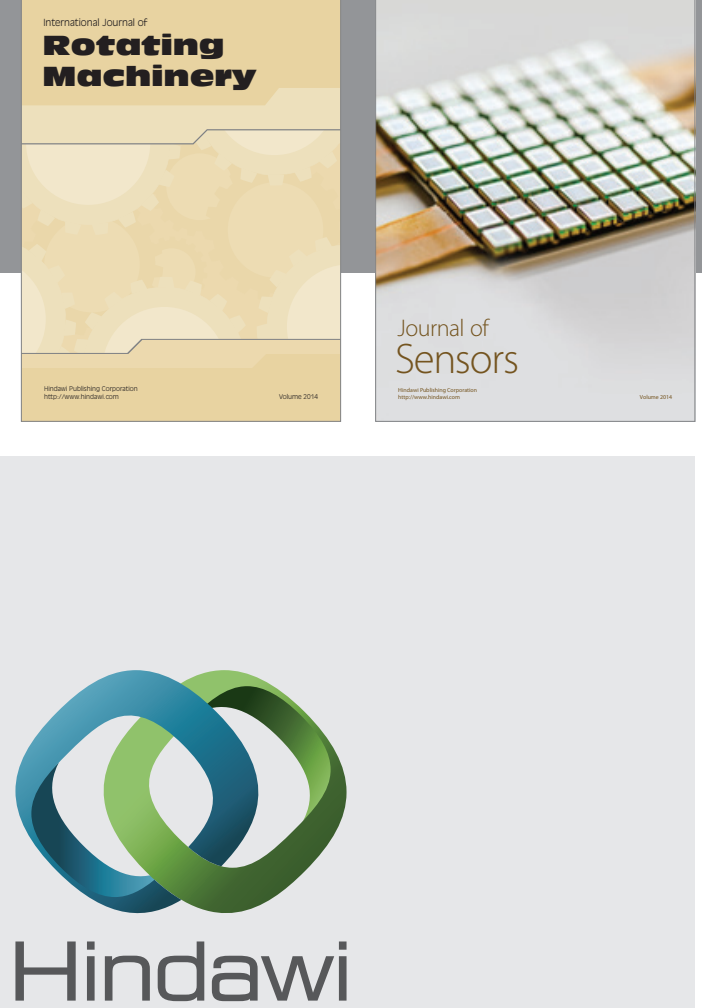

Submit your manuscripts at http://www.hindawi.com
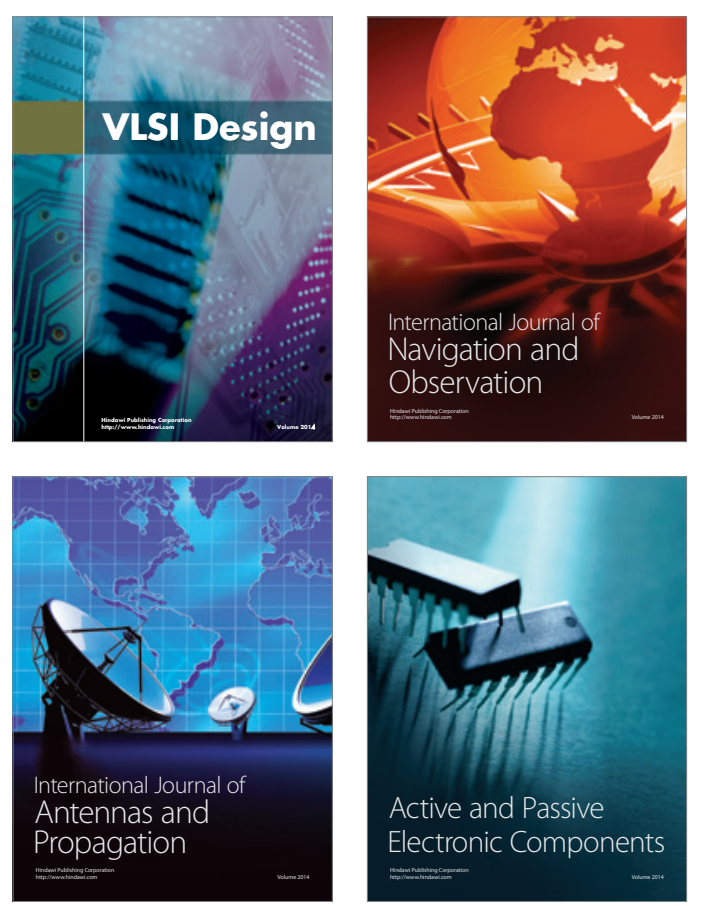
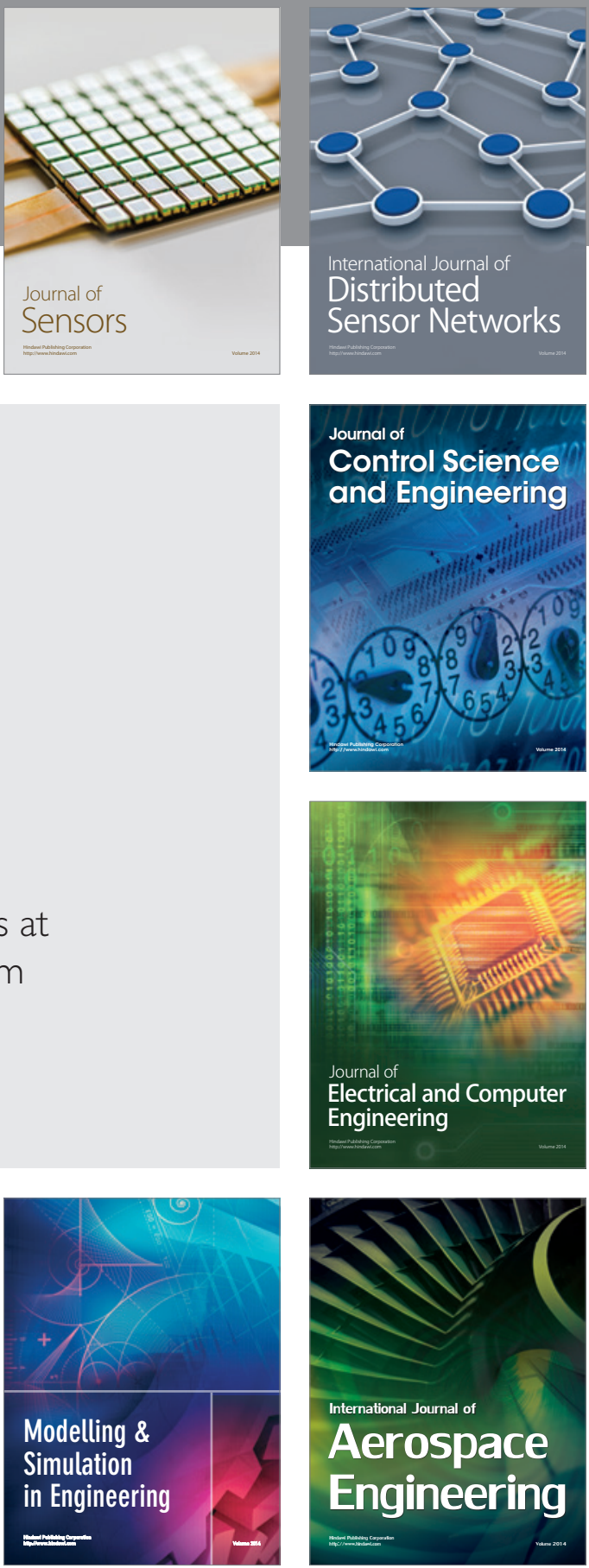

Journal of

Control Science

and Engineering
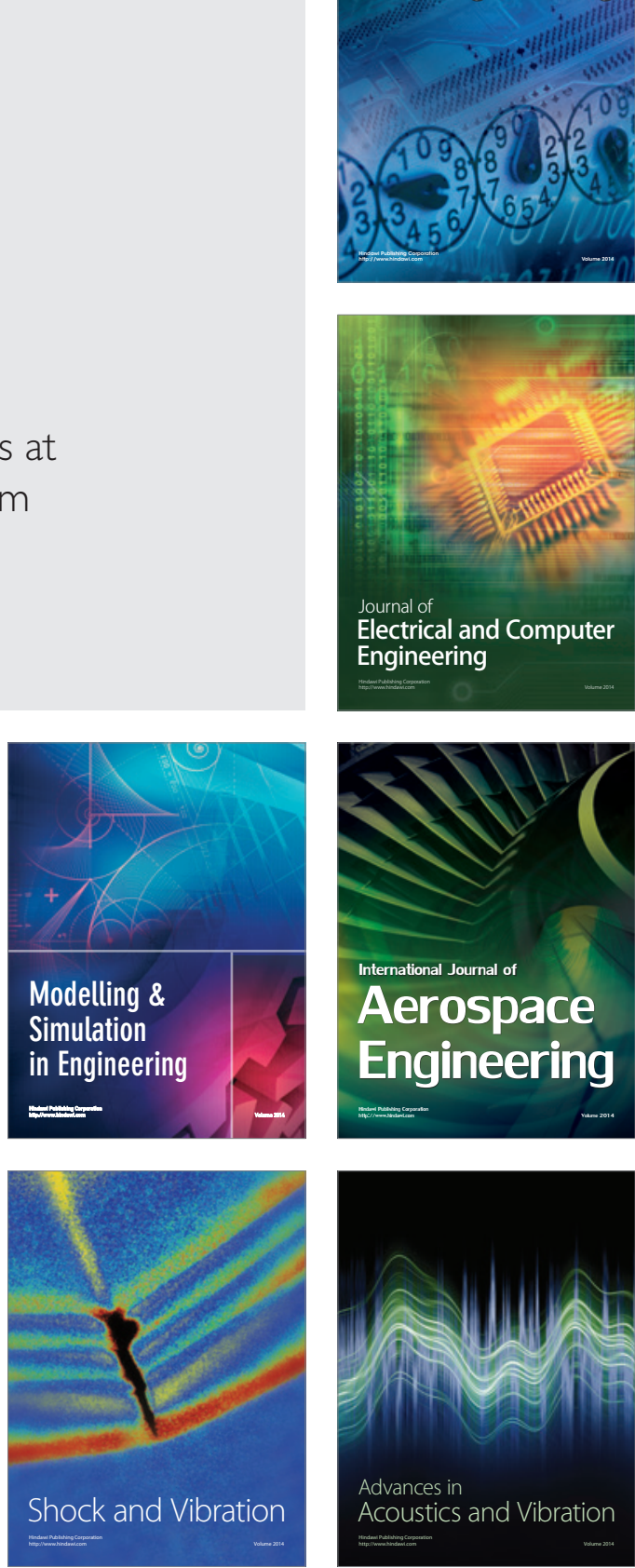